\title{
Postoperative Atrial Fibrillation in a Patient With Hypertrophic Obstructive Cardiomyopathy After Morrow Septal Myectomy May Evolve Into Lethal Ventricular Arrhythmia
}

\author{
Shiju Zhang ${ }^{\mathrm{a}}$, Qinjun Yu ${ }^{\mathrm{a}}$, Min Song ${ }^{\mathrm{a}}$, Yizhen Wei ${ }^{\mathrm{a}}$, Xiaohui Zhou ${ }^{\mathrm{a}}$, Qiang Guan ${ }^{\mathrm{a}}$, Shuiyun Wanga, b
}

\begin{abstract}
Postoperative atrial fibrillation (AF) is a common complication for patients undergoing cardiac surgery. However, in patients undergoing a Morrow septal myectomy procedure, postoperative AF can be disastrous. A 42-year-old man underwent a Morrow septal myectomy procedure for hypertrophic obstructive cardiomyopathy that was resistant to medical treatment. Doppler echocardiography demonstrated that the peak left ventricular outflow tract gradient dropped from 96 to $7 \mathrm{~mm} \mathrm{Hg}$ at rest after the procedure. The patient was doing well until the sudden onset of AF on the fifth night after surgery. Although medical conversion measures were promptly provided, the AF nonetheless evolved into ventricular fibrillation about 13 minutes after AF onset. The medical staff had to perform non-synchronized defibrillation to restore effective circulation. At 2 years after the procedure, the patient had not experienced AF recurrence and has recovered well.
\end{abstract}

Keywords: Hypertrophic obstructive cardiomyopathy; Postoperative atrial fibrillation; Morrow septal myectomy; Lethal ventricular arrhythmia; Cardiac surgery

\section{Introduction}

Although it has been documented that rapid atrial fibrillation (AF) can degenerate into ventricular fibrillation (VF) in patients with hypertrophic cardiomyopathy [1,2], for patients undergoing Morrow septal myectomy because of hypertrophic obstructive cardiomyopathy (HOCM), AF degeneration into VF has never been reported. This article demonstrates for the first time the danger of postoperative AF in an HOCM patient

Manuscript accepted for publication April 29, 2015

aState Key Laboratory of Cardiovascular Disease, Fuwai Hospital, National Center for Cardiovascular Diseases, Chinese Academy of Medical Science and Peking Union Medical College, Beijing 100730, China

${ }^{\mathrm{b}}$ Corresponding Author: Shuiyun Wang, State Key Laboratory of Cardiovascular Disease, Fuwai Hospital, National Center for Cardiovascular Diseases, Chinese Academy of Medical Science and Peking Union Medical College, Beijing 100730, China. Email: wangshuiyunsci@163.com

doi: http://dx.doi.org/10.14740/jmc2152w after surgery. Medical staff should be prepared for this situation in order to prevent disastrous consequences.

\section{Case Report}

A 42-year-old man was admitted to our facility because of HOCM refractory to $\beta$-blockers; his symptoms of exertional dyspnea and chest pain had become more frequent and even appeared after dinner from half year before the operation. The patient denied a family history of hypertrophic cardiomyopathy or AF. Physical examination found a degree 3/6 systolic murmur along the left sternal border, and echocardiography at rest revealed marked left ventricular hypertrophy, diffuse myocardial thickening, and impaired ventricular diastolic compliance. The patient's ventricular thickness was $25 \mathrm{~mm}$, left atrium diameter was $44 \mathrm{~mm}$, left ventricular end-diastolic diameter was $42 \mathrm{~mm}$, and ejection fraction was $78 \%$. The peak pressure gradient between the left ventricle and the ascending aorta was $96 \mathrm{~mm} \mathrm{Hg}$. The mitral valve showed mild to moderate regurgitation resulting from a systolic anterior motion of the anterior leaflet. ECG showed high voltage in the left ventricle and sinus bradycardia.

The patient underwent Morrow septal myectomy. The aortic cross-clamping time was $56 \mathrm{~min}$ and the extracorporeal time was $94 \mathrm{~min}$. After the operation, the left atrium diameter decreased to $35 \mathrm{~mm}$, the peak gradient of left ventricular outflow decreased from 96 to $9 \mathrm{~mm} \mathrm{Hg}$ at rest, and the subaortic septal thickness decreased from 25 to $13 \mathrm{~mm}$. The left ventricle's diameter and ejection fraction underwent no significant changes. ECG showed left bundle branch block.

During the first day after surgery, because of the patient showing slight hypotension, dobutamine was injected with a micropump. An oral $\beta$-blocker was prescribed to control the patient's heart rate the second day after surgery. Until the fifth day after the operation, the patient was doing well; however, on the fifth night, he began to experience AF with a ventricular heart rate of $140 \mathrm{bpm}$. The patient's systolic blood pressure dropped dramatically from 120 to $80 \mathrm{~mm} \mathrm{Hg}$ and he began to complain of dyspnea and palpation. Sublingual atenolol did not have any effect. During the 10 min after AF onset, the patient's ventricular heart rate increased from 140 to 170 bpm and his blood pressure dropped from 85 to $70 \mathrm{~mm} \mathrm{Hg}$. Norepinephrine and amiodarone were injected intravenously 
by micropump. About $13 \mathrm{~min}$ after onset, the AF evolved into ventricular tachycardia and then to VF. The patient lost consciousness and his pulse could not be found at the carotid artery. After confirming the stop of effective cardiac circulation, the medical staff applied non-synchronized defibrillation at once. VF ended and the patient's heart became temporarily pacemaker-dependent. His blood pressure returned to normal and he regained consciousness without any complications. Sinus rhythm was restored in $30 \mathrm{~min}$.

AF did not reoccur during the patient's time in the hospital. Two years after surgery, the patient is doing well and is without any symptoms of HOCM.

\section{Discussion}

As the most common type of arrhythmia after cardiac surgery, AF can contribute to increased morbidity and hospital stay [3, 4], although it does not significantly increase mortality [5]. For patients with HOCM who have undergone Morrow septal myectomy however, AF may incur fatal arrhythmia, as shown in this report. For these patients, hypertrophy of the cardiac muscle is diffuse and involves the entire left ventricle. Although the outflow tract has been thoroughly cleared, the diffuse hypertrophy still significantly impairs the left ventricle's compliance; therefore, the filling of the left ventricle is more dependent on the left atrium's contraction [6]. When the heart rate increases during AF, the heart's stroke volume decreases significantly. During this situation, sharply decreased blood pressure and coronary perfusion pressure combined with myocardial cell damage and edema after cardiopulmonary bypass will likely cause ventricular tachycardia.

Although there is no reliable measure for preventing postoperative AF, for these patients, some factors may be helpful. Temporary epicardial pacing leads should be used routinely and be sufficiently well-anchored in the ventricular myocardium during surgery. As shown in this case, a temporary pacemaker provided electrical stimulation to the patient's heart before the native sinus rhythm was restored after defibrillation. To maintain adequate preload and afterload, applying vasodilating agents and diuretics is commonly proscribed. Keeping the blood potassium at a relatively high level is helpful for stabilizing the electric action of the heart's cells. $\beta$-blockers should be routinely used, whereas positive inotropic agents are commonly contraindicated. Oral amiodarone immediately after surgery may be helpful, especially if the patient has a history of AF before the operation.
It is advisable to have a defibrillator at the bedside, considering the consequences of AF, as shown in this report. Because of a possibly significant drop in blood pressure, it may be necessary to use norepinephrine to raise blood pressure when using amiodarone to end AF. If the AF converts into VF and the patient loses consciousness, direct current non-synchronous defibrillation should be used immediately.

\section{Conclusion}

Postoperative AF after Morrow septal myectomy may evolve into ventricular arrhythmia. Special attention should be paid for it to prevent disastrous hemodynamic consequences.

\section{Conflict of Interests}

All authors declare that they have no any conflict of interests.

\section{References}

1. Cecchi F, Maron BJ, Epstein SE. Long-term outcome of patients with hypertrophic cardiomyopathy successfully resuscitated after cardiac arrest. J Am Coll Cardiol. 1989;13(6):1283-1288.

2. Stafford WJ, Trohman RG, Bilsker M, Zaman L, Castellanos A, Myerburg RJ. Cardiac arrest in an adolescent with atrial fibrillation and hypertrophic cardiomyopathy. J Am Coll Cardiol. 1986;7(3):701-704.

3. Maisel WH, Rawn JD, Stevenson WG. Atrial fibrillation after cardiac surgery. Ann Intern Med. 2001;135(12):10611073.

4. Banach M, Kourliouros A, Reinhart KM, Benussi S, Mikhailidis DP, Jahangiri M, Baker WL, et al. Postoperative atrial fibrillation - what do we really know? Curr Vasc Pharmacol. 2010;8(4):553-572.

5. Almassi GH, Schowalter T, Nicolosi AC, Aggarwal A, Moritz TE, Henderson WG, Tarazi R, et al. Atrial fibrillation after cardiac surgery: a major morbid event? Ann Surg. 1997;226(4):501-511; discussion 511-503.

6. Ommen SR, Thomson HL, Nishimura RA, Tajik AJ, Schaff HV, Danielson GK. Clinical predictors and consequences of atrial fibrillation after surgical myectomy for obstructive hypertrophic cardiomyopathy. Am J Cardiol. 2002;89(2):242-244. 